\title{
The Association between Dietary Inflammatory Index with Sleep Quality and Obesity among Iranian Female Students: A Cross-sectional Study
}

\author{
hadi bazyar ${ }^{1}$, ahmad zarejavid ${ }^{1}$, Hossein Bavi Behbahani ${ }^{1}$, SHIVAPPA NITIN $^{2}$, JAMES \\ HEBERT $^{2}$, Sara Khodaramhpour ${ }^{1}$, Sara Khaje Zadeh ${ }^{1}$, and vahideh aghamohammadi ${ }^{3}$ \\ ${ }^{1}$ Ahvaz Jondishapour University of Medical Sciences \\ ${ }^{2}$ University of South Carolina Arnold School of Public Health \\ ${ }^{3}$ Affiliation not available
}

November 30, 2020

\begin{abstract}
Background: Overweight, obesity and lack of sleep quality as inflammatory states are the common problems among college students and the Association of Dietary Inflammatory Index (DII) with these problems among this population is unknown. We aimed to evaluate the relationship of the DII with obesity and sleep quality among Iranian female students. Methods: The present cross-sectional study was conducted in 249 female college students. The Dietary Inflammatory Index (DII) was calculated using a valid and reliable 147-item food frequency questionnaire (FFQ). To assess sleep quality, Pittsburgh Sleep Quality Index (PSQI) was used. Odds Ratio (OR) and 95\% Confidence Intervals (CIs) were estimated for anthropometric indices and sleep quality according to DII score. Linear regression was used to estimate the relationship between DII score with sleep and anthropometric indices. Results: There was a significant association evident between DII and sleep quality( $>$ 5 is considered as poor quality of sleep); i.e., the odds ratios between DII quartile 2 vs 1 (unadjusted model: OR=0.33 (CI: $0.14-0.74), \mathrm{P}$ for trend $=0.002$; model 1 : the fully adjusted $\mathrm{OR}=0.31(\mathrm{CI}: 0.12-0.78), \mathrm{P}$ for trend $=0.005 ;$ model $2: \mathrm{OR}=0.30$ (CI:0.12-0.78), $\mathrm{P}$ for trend $=0.005)$ to quartile 4(unadjusted model: OR=1.13(CI: 0.45-2.80); model 1: OR=1.11(CI: $0.44-$ 2.79 ); model 2: $\mathrm{OR}=1.13(\mathrm{CI}: 0.44-2.87)$, $\mathrm{P}$ for trend $=0.005)$. Also, odds ratios increased significantly from quartile 2 to quartile 4 in all models for DII and sleep quality. According to the continuous score of DII, there was a significant positive association between DII and sleep quality in all 3 models: unadjusted, model 1, and model 2 (OR=1.21 (CI: 1.05-1.40), OR=1.21 (CI: 1.03-1.43), and $\mathrm{OR}=1.22$ (CI: 1.03-1.44), respectively. Conclusions: In this study, after removing the effect of confounding factors, participants in the highest quartile of DII score had significantly higher PSQI global score.
\end{abstract}

The Association betweenDietary Inflammatory Index with Sleep Quality and Obesity among Iranian Female Students: A Cross-sectional Study

Hadi Bazyar ${ }^{1,2}$, Ahmad Zare Javid ${ }^{2}$, Hossein Bavi Behbahani ${ }^{1,2}$, Nitin Shivappa ${ }^{3,4,5}$, James R Hebert ${ }^{3,4,5}$, Sara Khodaramhpour ${ }^{1}$, Sara Khaje Zadeh ${ }^{1}$, and Vahideh Aghamohammadi*6

1. Student Research Committee, Ahvaz Jundishapur University of Medical Sciences, Ahvaz, Iran.

2. Department of Nutrition, School of Allied Medical Sciences, Ahvaz Jundishapur University of Medical Science, Ahvaz, Iran.

3. Cancer Prevention and Control Program, University of South Carolina, Columbia, SC 29208, USA

4. Department of Epidemiology and Biostatistics, Arnold School of Public Health, University of South Carolina, Columbia, SC 29208, USA

5. Department of Nutrition, Connecting Health Innovations LLC, Columbia, SC, 29201, USA 
6. Department of Nutrition, Khalkhal University of Medical Science, Khalkhal, Iran.

* Correspondence to: Vahideh Aghamohammadi, Assistant Professor, Department of Nutrition, Khalkhal University of Medical Science, Khalkhal, Iran. Email address; v_agamohammadi@yahoo.com, Tel: $+989031512272$

\section{Abstract}

Background: Overweight, obesity and lack of sleep quality as inflammatory states are the common problems among college students and the Association of Dietary Inflammatory Index (DII) with these problems among this population is unknown. We aimed to evaluate the relationship of the DII with obesity and sleep quality among Iranian female students.

Methods: The present cross-sectional study was conducted in 249 female college students. The Dietary Inflammatory Index (DII) was calculated using a valid and reliable 147-item food frequency questionnaire (FFQ). To assess sleep quality, Pittsburgh Sleep Quality Index (PSQI) was used. Odds Ratio (OR) and 95\% Confidence Intervals (CIs) were estimated for anthropometric indices and sleep quality according to DII score. Linear regression was used to estimate the relationship between DII score with sleep and anthropometric indices.

Results: There was a significant association evident between DII and sleep quality $(>5$ is considered as poor quality of sleep); i.e., the odds ratios between DII quartile 2 vs 1 (unadjusted model: OR=0.33 (CI: $0.14-0.74), \mathrm{P}$ for trend $=0.002$; model 1 : the fully adjusted $\mathrm{OR}=0.31$ (CI: $0.12-0.78), \mathrm{P}$ for trend $=0.005$; model 2:OR=0.30 (CI:0.12-0.78), $\mathrm{P}$ for trend $=0.005)$ to quartile 4(unadjusted model: $\mathrm{OR}=1.13(\mathrm{CI}: 0.45-$ 2.80); model 1: $\mathrm{OR}=1.11(\mathrm{CI}: 0.44-2.79)$; model 2:OR=1.13(CI:0.44-2.87), $\mathrm{P}$ for trend $=0.005)$. Also, odds ratios increased significantly from quartile 2 to quartile 4 in all models for DII and sleep quality. According to the continuous score of DII, there was a significant positive association between DII and sleep quality in all 3 models: unadjusted, model 1 , and model $2(\mathrm{OR}=1.21$ (CI: 1.05-1.40), $\mathrm{OR}=1.21$ (CI: 1.03-1.43), and $\mathrm{OR}=1.22$ (CI: $1.03-1.44)$, respectively.

Conclusions: In this study, after removing the effect of confounding factors, participants in the highest quartile of DII score had significantly higher PSQI global score.

Keywords: Central Obesity, College Students, Dietary Inflammatory Index, Sleep Quality

\section{What's known}

- Overweight, obesity and lack of sleep quality as inflammatory states are the common problems among college students and the association of DII with these problems among this population is unknown.

- The Dietary Inflammatory Index (DIIß) was designed to assess the inflammatory potentials of the individuals 'diet.

- It was hypothesized that a diet with a higher inflammatory potential could be associated with obesity and poor sleep in Iranian female students.

What's new

- Those in DII quartile 1 had significantly more age, weight, BMI, WC, HC, and AVI, and good sleep quality compared to those in quartile 4 .

- According to continuous DII, in the unadjusted model, there was a significant negative association between DII score with weight, BMI, WC, HC, AVI, BAI, and conicity index.

- According to the continuous score of DII, there was a significant positive association between DII and sleep quality in all 3 models: unadjusted, model 1 , and model 2 (OR= 1.21 (CI: 1.05-1.40), OR=1.21 (CI: 1.03-1.43), and $\mathrm{OR}=1.22(\mathrm{CI}: 1.03-1.44)$, respectively.

Introduction 
Some studies are available on the alarming rate of overweight and obesity among college students ${ }^{1-4}$. The majority of college students do not regard the dietary and physical activity guidelines, indicating the necessity of prevention treatments and increased understanding of overweight in college students ${ }^{2}$. In addition to the high prevalence of overweight and obesity among college students, lack of sleep quality is also a prevalent occurrence among this population which has affected $10 \%$ to $50 \%$ of this population ${ }^{5,6}$ lack of sleep quality have profound effects on students' health (including increased risk of chronic diseases), quality of life, mood, and daytime function ${ }^{7,8}$. Lack of sleep quality similar to obesity, is associated with increased circulatory levels of pro-inflammatory markers and consequent insulin resistance and orexin decline ${ }^{9}$. Orexin is essential for the stability of sleep/wake states in various species, namely dog, mouse, and human ${ }^{10}$. Orexin knockout mice have exhibited frequent sudden collapses during the dark phase, the portion of the circadian rhythm during which there is the most time awake and spent in the activity. These attacks resemble human cataplexy attacks $^{11}$. Orexin neuron activity is suppressed by Tumor Necrosis Factor- alpha (TNF- $\alpha$ ) through this cytokine degrading the mRNA of orexin precursor in a time- and dose-dependent manner ${ }^{12,13}$.

Observational studies showed an independent association between the lack of sleep quality and elevated high-sensitivity C-Reactive Protein (hs-CRP), but this relationship was gender-specific in some studies ${ }^{14-17}$. Vgontzas and colleagues found that two hours of sleep reduction per night for seven nights led to increased TNF- $\alpha$ in healthy men but not women, but increased Interleukin 6 (IL-6) in both men and women ${ }^{18}$. In sum, sleep restriction leads to a systemic increase of the inflammatory mediators that may have prognostic significance for metabolic diseases.

Literature has supported diet represents an essential role in the modification and regulation of low-grade chronic inflammation ${ }^{19,20}$. So, it has been confirmed that Western dietary patterns have pro-inflammatory potential, and contrarily healthy dietary patterns such as the Mediterranean diet are related to antiinflammatory status ${ }^{21}$. Dietary patterns have been shown to influence inflammatory pathways; for instance, adherence to healthy dietary patterns such as the Mediterranean diet and/or national dietary guidelines can decrease inflammation and the $\mathrm{Mets}^{22}$. An umbrella meta-analysis of studies found promising evidence about the significant benefits of the Mediterranean diet in lowering body weight, Body Mass Index (BMI) and Waist Circumference (WC) as well as the improvement of lipid profile versus the control diets ${ }^{4}$. Okun et al. showed that young and healthy women with a poor sleep had more circulatory levels of C-Reactive Protein (CRP) compared to women with better sleep quality. The authors supposed that this pro-inflammatory status may increase the risk of various diseases that affect women, such as polycystic ovary syndrome and preeclampsia. However, they couldn't find any significant association between sleep duration and inflammatory markers ${ }^{23}$. The results of the National Health and Nutrition Examination Survey of (NHANES) 2005-2006 among 2051 adults ([?]20 years) indicated that having regular physical activity and not smoking and healthy eating and enough sleep were the two health behavior pairs related to lower systemic inflammation ${ }^{24}$. In this regard, the Dietary Inflammatory Index (DII $\left.{ }^{\circledR}\right)$ was designed to assess the inflammatory potentials of the individuals 'diet. It was proven that this index could predict inflammation-related health status in any population ${ }^{25}$. Two review studies concluded that the DII score appears to be a beneficial tool to assess the dietary inflammatory capacity and to perceive further the correlations between diet, inflammation, and chronic metabolic diseases such as obesity, diabetes, and Cardiovascular Diseases (CVD) ${ }^{26-28}$.

Overweight, obesity and lack of sleep quality as inflammatory states are the common problems among college students and the association of DII with these problems among this population is unknown ${ }^{28}$. It was hypothesized that a diet with a higher inflammatory potential could be associated with obesity and poor sleep in Iranian female students.

\section{Methods}

\section{Participants}

The present cross-sectional study was conducted in female college students who have studied at Ahvaz Jundishapur University of Medical Science, Iran. As regards, women's dietary and behavioral patterns are different. Also, hormonal changes in women may have different effects on their weight and sleep. So, it was 
decided to only examine women in this study. In this study, cluster sampling was performed and 249 students aged 18-35 years from a population of 300 candidates were surveyed in this research. Inclusion criteria were the willingness to participate in the study and the age range of 18 to 35 years. We excluded 51 students who met the exclusion criteria (included unwillingness to participate in the study, incomplete demographic or anthropometric data, not responding to more than 35 food items in the Food Frequency Questionnaire (FFQ), pregnancy, breastfeeding, smoking, consuming alcohol, and any drugs and supplements, having an eating disorder, diabetes, cardiovascular disease, kidney disorders, thyroid, digestive and respiratory diseases, cancer, adherence to the specific diets, total energy intake less than $800 \mathrm{kcal}$ and more than $4200 \mathrm{kcal}$, and having a weight loss surgery in the last year) were excluded from the study.

\section{Sample size}

The sample size was determined based on BMI (mean=25.1 and $\mathrm{SD}=5.1)$ as the primary outcome obtained from the study by Kord Varkaneh et al. ${ }^{29}$ in women referred to health centers affiliated to Tehran University of Medical Sciences, Iran. The sample size was computed using the following formula: $\mathrm{N}=\left[\left(\mathrm{z}_{1-\alpha / 2}\right)^{2} \times \mathrm{sd}^{2}\right] / \mathrm{d}^{2}(\alpha=$ 0.05 , confidence level of $95 \%$ and $\mathrm{d}=2 \%$ ) as 250 subjects. Considering the withdrawal rate of $20 \%, 300$ subjects were recruited.

\section{Ethical approval}

The participants who met the inclusion criteria were fully informed about the study's protocol. The study protocol was approved by the Ethics Committee of Ahvaz Jundishapur University of Medical Science (IR.AJUMS.REC.1397.817). Each subject signed the written informed consent.

\section{Anthropometric and physical activity assessment}

Bodyweight and height were measured using a Seca scale with an accuracy of 100 gr and a Seca stadiometer with an accuracy of $0.5 \mathrm{~cm}$, respectively; and then, BMI was calculated as the body weight $(\mathrm{kg})$ divided by the square of height $(\mathrm{m})$. Waist circumference (WC) was measured in standing position using a tape with an accuracy of $1.0 \mathrm{~cm}$ above the iliac crest, just below the lowest rib margin at the end of the normal expiration. ${ }^{30}$ For hip circumference (HC) determination, the tape was put around the point with the maximum circumference over the buttocks ${ }^{31}$. The other anthropometric indices, such as abdominal volume index $(\mathrm{AVI})$, conicity index $(\mathrm{CI})$, and BAI $(\mathrm{kg} / \mathrm{m} 2)$ were calculated using the following formulas: ${ }^{32-34}$

$$
\begin{aligned}
\text { AVI } & =\frac{\left[2 \mathrm{~cm} \times(\text { waist }(\mathrm{cm}))^{2}+0.7 \mathrm{~cm} \times(\text { waist }(\mathrm{cm})-\text { hip }(\mathrm{cm}))^{2}\right]}{1000}, \\
\text { BAI } & =\frac{\text { hip circumference }(\mathrm{cm})}{\text { height }(\mathrm{m})^{1.5}}-18, \\
\mathrm{CI} & =\frac{\text { waist circumference }(\mathrm{m})}{0.109 \sqrt{\text { weight }(\mathrm{kg}) / \text { height }(\mathrm{m})}} .
\end{aligned}
$$

To evaluate the rate of the physical activity of individuals, the short form of the International Physical Activity Questionnaire (IPAQ) was used via interviewing, and the results were represented as metabolic equivalent hours per week (METs hr/wk) ${ }^{35,36}$. Moreover, the socio-demographic characteristics of participants were acquired through a questionnaire.

\section{Dietary assessment}

Trained dietitians obtained the usual food intake through a structured interview. A valid and reliable 147item semi-quantitative FFQ with standard servings was applied to determine typical food intakes. ${ }^{37,38}$ The intake frequency of each food item was asked on a daily, weekly, or monthly basis during the past year and 
converted to the gram. Total energy and nutrient intake were then calculated using Nutritionist IV software (the Hearst Corporation, San Bruno, CA) as modified for Iranian foods.

\section{DII calculation}

A complete description of DII has been provided by Shivappa et al. ${ }^{25}$ The index is based on 1943 articles published since 1950 to 2010 and measures the effects of 45 dietary parameters on six inflammatory biomarkers. (IL-1 $\beta$, IL-6, TNF- $\alpha$, CRP, IL-4, and IL-10). In fact, each one of these dietary factors received a negative score (-1) if its impact was anti-inflammatory (significantly increased IL-4 or IL-10, or reduced IL-1 $\beta$, IL-6, TNF- $\alpha, \mathrm{CRP})$, a positive score $(+1)$ if its impact was pro-inflammatory and 0 if no significant changes was observed in biomarkers. To calculate the DII score, each study participant's dietary data was first linked to the global database that provided a robust estimate of a mean intake and a standard deviation for each of the 45 food parameters. In this study, of the 45 possible food items in the DI list, dietary intakes of 30 food parameters including energy, protein, carbohydrates, fiber, total fat, saturated, monounsaturated and, polyunsaturated fatty acids, omega 3 and omega 6 fatty acids, trans fats, cholesterol, vitamins A, beta carotene, E, D, C, B1, B2, B3, B6, B12, folate, zinc, selenium, magnesium, Iron (Fe), garlic, onion, and caffeine, were applied to compute the DII. To obtain the z-score, the mean global for each food parameter was subtracted from the actual intake of each food parameters and then divided by its standard deviation. To minimize the effect of "right skewing," Z scores were converted to the centered proportion score as multiplied by 2 and subtracting 1 . To acquire the food parameter-specific DII score, the final value was multiplied by its respective overall food parameter score. Subsequently, the DII score was computed by summing all food parameter-specific DII scores. The higher DII score, the more pro-inflammatory diet, while a more negative value indicated a more anti-inflammatory diet. The theoretical maximum range of DII scores was from about -8.87 to about $+7.98^{21,25}$.

\section{Assessment of sleep quality}

To assess sleep quality, we applied the 18 self -reported items in Pittsburgh's sleep quality index (PSQI)

26. The PSQI questionnaire was generated by Buysse et al. for assessment of sleep quality and detection of sleep disturbances and involved 7 subscales, including subjective sleep quality, sleep latency, sleep duration, sleep efficiency, sleep disturbances, use of sleep medication and daytime dysfunction. Scales of each subscale is ranged from 0 to 3 . Moreover, the total score of these 7 parts ranges from 0 to 21. The global PSQI score over 5 is considered as poor quality of sleep. Participants were asked to respond their bed time and wake time to the nearest hour and the times were then used to compute duration of sleep ${ }^{39}$. Validity and reliability of this questionnaire have been investigated in $\operatorname{Iran}(\alpha=0.83 \text { and correlation coefficient }=0.88)^{40}$.

\section{Statistical analysis}

All statistical analyses were fulfilled using IBM SPSS Statistics software (Version 24) (IBM SPSS Statistics, Armonk, USA). The normality of variables was established using the Kolmogorov- Smirnov test. DII scores were categorized into quartiles. The differences in variables across the quartiles of DII were detected using One-way ANOVA. A comparison of the mean intake of nutrients with global mean intake per day was performed using one-sample t-test. To compare the categorical variables across quartiles, the Chi-squared test was applied. Linear regression analysis was used in 3 models (Model 0, linear regression analysis without adjustment; Model I, linear regression analysis with adjustment for energy intake; Model II, linear regression analysis with correction for age, energy intake, physical activity, and education) for determination of the Association between DII score as the independent variable with anthropometric variables and PSQI continuous score as the dependent variables. Moreover, the odds ratio (OR) and 95\% confidence intervals (CIs) were estimated using multivariable logistic regression in 3 different models, including Model 0: unadjusted, Model 1: adjusted for energy intake and Model 2: adjustment for age, education, physical activity, energy intake. Also, p-values for linear trends was used in logistic regression to compare the OR between different quarters (Q1to Q4). The lower quartile of DII score (Q1) was used as the reference category. A p-value of less than 0.05 was regarded to be statistically significant.

\section{Results}




\section{The characteristics of participants across quartiles of DII}

The participants' characteristics across the quartiles of DII have been shown in Table 1. The differences in physical activity, BMI categories, education, BAI, conicity index, and PSQI categories were not significant between DII quartiles (p [?] 0.05). Those in DII quartile 1 had significantly more age, weight, BMI, WC, $\mathrm{HC}$, and AVI, and good sleep quality compared to those in quartile $4(\mathrm{p}<0.05)$.

\section{Dietary intake of participants according to quartiles of DII}

The mean \pm SD of energy, macronutrient, and micronutrient intake across quartiles of DII are presented in (Table2). No significant differences was observed in the intake of Vit D, onion, trans fat, and cholesterol between DII quartiles ( $\mathrm{p}$ [?] 0.05). While the differences in the other 26 food intake variables were statistically significant $(\mathrm{p}<0.05)$. Participants in quartile 4 had higher intakes of total fat, saturated fatty acid (SFA), and monounsaturated fatty acid (MUFA) in comparison to those in quartile $1(\mathrm{p}<0.001)$. But, the intake of polyunsaturated fatty acid (PUFA) and fiber were significantly lower in quartile 4 than in quartile 1 (p $<0.001$ ) (Table2). Comparison of the mean intake of nutrients with global mean intake per day ${ }^{41}$ based on one sample t-test indicated the significant difference in the intake of the majority of food parameters $(\mathrm{p}<$ 0.05) except for thiamin, MUFA, total fat, and energy intake (supplementary Table1).

\section{Relationship between DII score with anthropometric indices and PSQI score}

The Association between DII score with anthropometric variables including weight, BMI, WC, HC, AVI, BAI, and conicity index and PSQI score is illustrated in Table 3. According to continuous DII, in the unadjusted model, there was a significant negative association between DII score with weight, BMI, WC, HC, AVI, BAI, and conicity index ( $\mathrm{p}$ for all $<0.05$ ); however, in Model 1 and after adjustment for energy intake this negative correlation disappeared and a positive correlation was found, but it was not statistically significant for all anthropometric variables (p [?] 0.05) (Table 3).

Regarding the Association between DII score (continuous DII) as an independent variable and PSQI continuous score as dependent variables, in the unadjusted model, there was no significant association between these two variables $(\beta$-Coefficients $=0.18$ and $p=0.1)$. While, in adjusted models, there was a significant positive association between DII and sleep score (model 1: $\beta$-Coefficients $=0.29$ and $\mathrm{p}=0.02$, model 2 : $\beta$-Coefficients $=0.30$ and $\mathrm{p}=0.02$ ). Also, according to quartiles of the DII, there was a significant positive association between DII and sleep score in all 3 models, unadjusted, model 1 , and model 2 (unadjusted: $\beta$-Coefficients $=0.41$ and $\mathrm{p}=0.04$, model $1: \beta$-Coefficients $=0.61$ and $\mathrm{p}=0.008$, and model $2: \beta$-Coefficients $=0.60$ and $\mathrm{p}=0.01$, respectively ) (Table 3 ).

Odds ratios (95\% CI) for obesity and sleep quality, according to quartiles of DII, are presented in Table 4. There was a significant difference in the odds ratios between the quartile 1 (the reference quartile) (unadjusted model: $\mathrm{OR}=0.33$ (CI: 0.14-0.74), $\mathrm{P}$ for trend $=0.002$; model 1 : $\mathrm{OR}=0.31(\mathrm{CI}: 0.12-0.78), \mathrm{P}$ for trend $=0.005$; model $2: \mathrm{OR}=0.30(\mathrm{CI}: 0.12-0.78), \mathrm{P}$ for trend $=0.005)$ to quartile 4(unadjusted model: $\mathrm{OR}=1.13$ (CI: 0.45-2.80); model 1: $\mathrm{OR}=1.11(\mathrm{CI}: 0.44-2.79)$; model 2:OR=1.13(CI:0.44-2.87), $\mathrm{P}$ for trend $=$ 0.005). Also, odds ratios increased significantly from quartile 2 to quartile 4 in all models for DII and sleep quality.

On the other hand, according to the continuous score of DII, there was a significant positive association between DII and sleep quality in all 3 models: unadjusted, model 1, and model 2 (OR=1.21 (CI: 1.05-1.40), $\mathrm{OR}=1.21$ (CI: 1.03-1.43), and $\mathrm{OR}=1.22(\mathrm{CI}: 1.03-1.44)$, respectively $($ Table 4$)$.

\section{Discussion}

In the present study, the Association of DII score with obesity indexes and sleep quality was assessed in Iranian female college students which indicated no significant association between DII score and obesity. However, a significant association was observed between the DII and PSQI score.

Consistent with our findings, the study by Sen et al. did not show any significant associations between DII and obesity $(\mathrm{OR}=1.4,95 \% \mathrm{CI}-0.8-2.3, \mathrm{p}=0.23) .{ }^{42}$ Moreover, the findings from the SU.VI.MAX study with 
3726 participants indicated no significant association between WC and DII score $(\mathrm{p}=0.45) .{ }^{43}$ In another cross-sectional survey among the Luxembourg population (ORISCAV-LUX), no significant associations was observed in the Association between DII score and WC, which was in line with our findings. ${ }^{44}$ Moreover, a cross-sectional study among 464 police officers indicated that DII has no association with WC as a Mets component. ${ }^{45}$ Sokol et al. showed a significant association of DII with waist components only in males, not in females. ${ }^{46}$ In contrast to our findings, in some other research studies that were carried out in Iran, ${ }^{47}$ and Spain (PREDIMED' study and SUN cohort study), ${ }^{48,49}$ a significant association between DII and obesity or Mets components were detected. Lack of significant Association of the DII score with WC and BMI in the present study may be related to the sample size, type of research (cross-sectional), the numbers of food items in the calculation of DII and a small mean of BMI compared to studies that have observed a significant association. In the present study, a significant association was observed between the DII and PSQI score. In the recent study by Lopez et al., it was shown that the DII score was only positively associated with daytime sleepiness as a component of PSQI $(\beta=0.47$, p value $<0.05) .{ }^{50}$ In the study by Godos et al. participants in the highest quartile of DII score were more likely to have a poor sleep (OR $=0.49,95 \%$ CI: $0.31-0.78)$. Moreover, the authors demonstrated that high adherence to the Mediterranean diet as an anti-inflammatory dietary pattern, could improve sleep quality in Italian adults through the direct and indirect effects on health and body weight status, respectively. ${ }^{51}$ Bilgic et al. showed that increased malnutrition-inflammation score was significantly associated with the presence of depression, sleep disorders, and poor quality of life ${ }^{52}$.

The evidence from studies represented that that poor sleep was associated with chronic, low-grade inflammation status. Short and long sleep duration was associated with increased inflammation markers, and sleep disruption may be related to diabetes via a mechanism of low-grade systemic inflammation ${ }^{53}$. Several studies suggested that a decrease in the number of hypocretin-containing neurons may be caused by an inflammatory- or immune-mediated process ${ }^{54-56}$. Hypocretin cell loss was observed in most patients with sleep disorder narcolepsy ${ }^{54}$.

Gordon-Dseagu et al. examined the Association between sleep variables and 891 fasting plasma metabolites among the participants from the Dietary Approaches to Stop Hypertension (DASH) trial. They observed that sleep parameters was associated with metabolites and pathways which had a relationship with inflammation and oxidative stress, including erythrulose (advanced glycation end-product) (positive Association) and several $\gamma$-glutamyl pathway metabolites, including CMPF (fatty acid, dicarboxylate), isovalerate (valine, leucine and isoleucine and fatty acid metabolism) and HWESASXX (polypeptide) (inverse association). ${ }^{57}$ The mechanisms about the Association between the DII score and health may be linked to the primary role of several dietary components on systemic inflammatory pathways and gut microbiota. ${ }^{58,59}$ It has been shown that low intake of vegetables and unhealthy eating habits were associated with poor sleep quality; vegetables are important sources of vitamins and polyphenols, which can lead to reduce pro-inflammatory response and oxidative damage, and represent neuroprotective effects. ${ }^{60}$ Omega 3 fatty acids and MUFA have known as anti-inflammatory properties. It was found in some studies regarding both Docosahexaenoic acid and the Docosahexaenoic acid: Arachidonic acid ratio affected sleep quality and inflammation ${ }^{61}$. In contrast, high intake of meat products and refined carbohydrates have been related to an increase in pro-inflammatory response. ${ }^{60}$ Regarding the influence of the gut microbiota on inflammation, it could be stated that there was a large data of various studies suggesting a mechanistic link between gut-derived inflammatory response and neurodegeneration via the pathways including modulation of plasma levels of lipopolysaccharide, the inflammasome, type I interferon, and nuclear factor kappa B cells (NF-KB) ${ }^{62}$ It should be noted that the present study's participants had less intake of foods impacting the availability of tryptophan, and its conversion to serotonin and melatonin such as protein and vitamins B group compared to global mean intake. ${ }^{63}$

\section{Limitations and Strengths}

The present study had some limitations, as well. In addition to the lack of generalizability, this study had a cross-sectional approach; then, no causal relationships could be established among variables. Moreover, polysomnographic recordings were not applied due to restrictions in funding. Despite these limitations, this 
was the first study that evaluated the associations of the DII scores with obesity and sleep quality in young Iranian female. Moreover, we examined the Association between DII score and anthropometric variables and PSQI score using linear regression analysis and multivariable logistic regression in 3 different models.

\section{Conclusions}

In conclusion, the findings of the present study showed that higher DII score was significantly associated with poor sleep (PSQI $>5$ ). After adjustment, participants in the highest quartile of DII score had significantly higher PSQI global score. There was no significant association between DII score and obesity. Our findings underscored the importance of eating an anti-inflammatory diet for good sleep. Future studies with prospective designs, larger sample size and considering more food parameters in the computation of the DII score, should be carried out to clarify this relationship.

\section{Acknowledgements}

The authors thank from the students who have collaborate with us sincerely to the end of study.

\section{Funding}

This study was financially supported by Student Research Committee of Ahvaz Jundishapur University of Medical Sciences (Grant's number: 97s34).

\section{Data Sharing Statement}

The datasets used and/or analyzed during the current study are available from the corresponding author on reasonable request.

\section{Authors' Contributions}

All authors made a significant contribution to the work reported, whether that is in the conception, study design, execution, acquisition of data, analysis and interpretation, or in all these areas; took part in drafting, revising or critically reviewing the article; gave final approval of the version to be published; have agreed on the journal to which the article has been submitted; and agree to be accountable for all aspects of the work.

\section{Ethics approval and consent to participate}

The participants who met the inclusion criteria were fully informed about the study's protocol. The study protocol was approved by the Ethics Committee of Ahvaz University of Medical Sciences (IR.AJUMS.REC.1397.817). Each subject signed the written informed consent. This study was conducted in accordance with the Declaration of Helsinki.

Disclosure: Dr. James R. Hébert owns controlling interest in Connecting Health Innovations LLC (CHI), a company that has licensed the right to his invention of the dietary inflammatory index (DII $\left.{ }^{\circledR}\right)$ from the University of South Carolina in order to develop computer and smart phone applications for patient counselling and dietary intervention in clinical settings. Dr. Nitin Shivappa is an employee of CHI.

\section{References}

1. Zar A, KARAN KP, AHMADI MA. Prevalence of obesity and overweight among female students of Shiraz University of Medical Sciences and its association with physical fitness factors. 2017.

2. Al-Kilani H, Waly M, Yousef R. Trends of obesity and overweight among college students in Oman: A cross sectional study. Sultan Qaboos University Medical Journal. 2012;12(1):69.

3. AlMajed HT, AlAttar AT, Sadek AA, et al. Prevalence of dyslipidemia and obesity among college students in Kuwait. Alexandria Journal of Medicine. 2011;47(1):67-71.

4. Dinu M, Pagliai G, Casini A, Sofi F. Mediterranean diet and multiple health outcomes: an umbrella review of meta-analyses of observational studies and randomised trials. European journal of clinical nutrition. 2018;72(1):30-43. 
5. Moo-Estrella J, Pérez-Benítez H, Solís-Rodríguez F, Arankowsky-Sandoval G. Evaluation of depressive symptoms and sleep alterations in college students. Archives of medical research.2005;36(4):393-398.

6. Curcio G, Ferrara M, De Gennaro L. Sleep loss, learning capacity and academic performance. Sleep medicine reviews. 2006;10(5):323-337.

7. Dinis J, Bragança M. Quality of sleep and depression in college students: a systematic review. Sleep Science. 2018;11(4):290.

8. Krystal AD, Thakur M, Roth T. Sleep disturbance in psychiatric disorders: effects on function and quality of life in mood disorders, alcoholism, and schizophrenia. Annals of Clinical Psychiatry.2008;20(1):39-46.

9. Clark IA, Vissel B. Inflammation-sleep interface in brain disease: TNF, insulin, orexin. Journal of neuroinflammation.2014;11(1):51.

10. Mieda M, Sakurai T. Integrative physiology of orexins and orexin receptors. CNS $\&$ Neurological Disorders-Drug Targets (Formerly Current Drug Targets-CNS 83 Neurological Disorders). 2009;8(4):281-295.

11. Chemelli RM, Willie JT, Sinton CM, et al. Narcolepsy in orexin knockout mice: molecular genetics of sleep regulation. Cell.1999;98(4):437-451.

12. Zhan S, Cai G-Q, Zheng A, et al. Tumor necrosis factor-alpha regulates the Hypocretin system via mRNA degradation and ubiquitination.Biochimica et Biophysica Acta (BBA)-Molecular Basis of Disease.2011;1812(4):565-571.

13. Clark IA, Vissel B. Inflammation-sleep interface in brain disease: TNF, insulin, orexin. Journal of neuroinflammation.2014;11(1):1-11.

14. Roca GQ, Redline S, Claggett B, et al. Sex-specific association of sleep apnea severity with subclinical myocardial injury, ventricular hypertrophy, and heart failure risk in a community-dwelling cohort: The Atherosclerosis Risk in Communities-Sleep Heart Health Study. Circulation. 2015;132(14):1329-1337.

15. Lee EE, Ancoli-Israel S, Eyler LT, et al. Sleep disturbances and inflammatory biomarkers in schizophrenia: focus on sex differences. The American Journal of Geriatric Psychiatry. 2019;27(1):21-31.

16. Punjabi NM, Beamer BA. C-reactive protein is associated with sleep disordered breathing independent of adiposity. Sleep.2007;30(1):29-34.

17. Irwin MR, Olmstead R, Carroll JE. Sleep disturbance, sleep duration, and inflammation: a systematic review and meta-analysis of cohort studies and experimental sleep deprivation. Biological psychiatry. 2016;80(1):40-52.

18. Vgontzas AN, Zoumakis E, Bixler EO, et al. Adverse effects of modest sleep restriction on sleepiness, performance, and inflammatory cytokines. The Journal of Clinical Endocrinology $\&$ Metabolism.2004;89(5):21192126.

19. Giugliano D, Ceriello A, Esposito K. The effects of diet on inflammation: emphasis on the metabolic syndrome. Journal of the American College of Cardiology. 2006;48(4):677-685.

20. Galland L. Diet and inflammation. Nutrition in Clinical Practice. 2010;25(6):634-640.

21. Wirth MD, Hébert JR, Shivappa N, et al. Anti-inflammatory Dietary Inflammatory Index scores are associated with healthier scores on other dietary indices. Nutrition research. 2016;36(3):214-219.

22. Ahluwalia N, Andreeva VA, Kesse-Guyot E, Hercberg S. Dietary patterns, inflammation and the metabolic syndrome. Diabetes $\mathcal{E}$ metabolism. 2013;39(2):99-110.

23. Okun ML, Coussons-Read M, Hall M. Disturbed sleep is associated with increased C-reactive protein in young women. Brain, behavior, and immunity. 2009;23(3):351-354. 
24. Loprinzi PD. Health behavior combinations and their association with inflammation. American Journal of Health Promotion.2016;30(5):331-334.

25. Shivappa N, Steck SE, Hurley TG, Hussey JR, Hébert JR. Designing and developing a literature-derived, population-based dietary inflammatory index. Public health nutrition. 2014;17(8):1689-1696.

26. Masaad AA, Yusuf AM, Shakir AZ, et al. Sleep quality and Dietary Inflammatory Index among university students: a cross-sectional study.Sleep and Breathing. 2020:1-9.

27. Tabung FK, Steck SE, Zhang J, et al. Construct validation of the dietary inflammatory index among postmenopausal women. Annals of epidemiology. 2015;25(6):398-405.

28. Ruiz-Canela M, Bes-Rastrollo M, Martínez-González MA. The role of dietary inflammatory index in cardiovascular disease, metabolic syndrome and mortality. International journal of molecular sciences.2016;17(8):1265.

29. Vivekananda M, Vandana K, Bhat K. Effect of the probiotic Lactobacilli reuteri (Prodentis) in the management of periodontal disease: a preliminary randomized clinical trial. Journal of oral microbiology. 2010;2(1):5344.

30. Haidari F, Aghamohammadi V, Mohammadshahi M, Ahmadi-Angali K. Effect of whey protein supplementation on levels of endocannabinoids and some of metabolic risk factors in obese women on a weight-loss diet: a study protocol for a randomized controlled trial. Nutrition journal.2017;16(1):70.

31. Ahmad N, Adam SIM, Nawi AM, Hassan MR, Ghazi HF. Abdominal obesity indicators: Waist circumference or waist-to-hip ratio in Malaysian adults population. International journal of preventive medicine.2016;7.

32. Owiredu WK, Osei-Yeboah J, Aryee C, Owusu-Dabo E, Laing EF, Owusu IK. Gender Specific Predictive Performance and Optimal Threshold of Anthropometric Indices for the Prediction of Hypertension among a Ghanaian Population in Kumasi. Asian Journal of Medicine and Health. 2017:1-10.

33. Hadi S, Momenan M, Cheraghpour K, et al. Abdominal volume index: a predictive measure in relationship between depression/anxiety and obesity. African Health Sciences. 2020;20(1):257-265.

34. Abolnezhadian F, Hosseini SA, Alipour M, et al. Association Metabolic Obesity Phenotypes with Cardiometabolic Index, Atherogenic Index of Plasma and Novel Anthropometric Indices: A Link of FTO-rs9939609 Polymorphism. Vascular Health and Risk Management.2020;16:249-256.

35. Craig C, Marshall A, Sjostrom M, et al. International Physical Activity Questionnaire-Short Form. 2017.

36. Bazyar H, Ahmadi A, Javid AZ, Irani D, Sartang MM, Haghighizadeh MH. The association between dietary intakes and stone formation in patients with urinary stones in Shiraz. Medical journal of the Islamic Republic of Iran. 2019;33:8.

37. Nikniaz L, Tabrizi J, Sadeghi-Bazargani H, Farahbakhsh M, Tahmasebi S, Noroozi S. Reliability and relative validity of short-food frequency questionnaire. British Food Journal. 2017.

38. Sajadi KP, Sedighian F, ALAODDOLEHEI H, Rekabpour KB. Food consumption frequency in Paramedical students of Babol University of Medical Sciences living in dormitories and other places. 2018.

39. Buysse DJ, Reynolds CF, Monk TH, Berman SR, Kupfer DJJPr. The Pittsburgh Sleep Quality Index: a new instrument for psychiatric practice and research. 1989;28(2):193-213.

40. Ahmadi S, Khankeh H, Mohammadi F, Khoshknab F, Reza Soltani P. The effect of sleep restriction treatment on quality of sleep in the elders. Iranian Journal of Ageing. 2010;5(2):0-0.

41. Mahan LK, Raymond JL. Krause's food \&s the nutrition care process-e-book. Elsevier Health Sciences; 2016. 
42. San KMM, Fahmida U, Wijaksono F, Lin H, Zaw KK, Htet MK. Chronic low grade inflammation measured by dietary inflammatory index and its association with obesity among school teachers in Yangon, Myanmar.Asia Pacific journal of clinical nutrition. 2018;27(1):92.

43. Neufcourt L, Assmann K, Fezeu L, et al. Prospective association between the dietary inflammatory index and metabolic syndrome: Findings from the SU. VI. MAX study. Nutrition, Metabolism and Cardiovascular Diseases. 2015;25(11):988-996.

44. Alkerwi Aa, Shivappa N, Crichton G, Hébert JR. No significant independent relationships with cardiometabolic biomarkers were detected in the Observation of Cardiovascular Risk Factors in Luxembourg study population. Nutrition research. 2014;34(12):1058-1065.

45. Wirth M, Burch J, Shivappa N, et al. Association of a dietary inflammatory index with inflammatory indices and the metabolic syndrome among police officers. Journal of occupational and environmental medicine/American College of Occupational and Environmental Medicine.2014;56(9):986.

46. Sokol A, Wirth MD, Manczuk M, et al. Association between the dietary inflammatory index, waist-to-hip ratio and metabolic syndrome. Nutrition research. 2016;36(11):1298-1303.

47. Nikniaz L, Nikniaz Z, Shivappa N, Hébert JR. The association between dietary inflammatory index and metabolic syndrome components in Iranian adults. Primary care diabetes. 2018;12(5):467-472.

48. Ruiz-Canela M, Zazpe I, Shivappa N, et al. Dietary inflammatory index and anthropometric measures of obesity in a population sample at high cardiovascular risk from the PREDIMED (PREvencion con DIeta MEDiterranea) trial. British journal of nutrition.2015;113(6):984-995.

49. Ramallal R, Toledo E, Martínez JA, et al. Inflammatory potential of diet, weight gain, and incidence of overweight/obesity: The SUN cohort.Obesity. 2017;25(6):997-1005.

50. Lopes TV, Borba ME, Lopes RV, et al. Association between the inflammatory potential of the diet and sleep parameters in sleep apnea patients. Nutrition. 2019;66:5-10.

51. Godos J, Ferri R, Caraci F, et al. Adherence to the mediterranean diet is associated with better sleep quality in italian adults. Nutrients. 2019;11(5):976.

52. Bilgic A, Akgul A, Sezer S, Arat Z, Ozdemir FN, Haberal M. Nutritional status and depression, sleep disorder, and quality of life in hemodialysis patients. Journal of Renal Nutrition.2007;17(6):381-388.

53. Shan Z, Ma H, Xie M, et al. Sleep duration and risk of type 2 diabetes: a meta-analysis of prospective studies. Diabetes care.2015;38(3):529-537.

54. Gerashchenko D, Shiromani PJ. Effects of inflammation produced by chronic lipopolysaccharide administration on the survival of hypocretin neurons and sleep. Brain research. 2004;1019(1-2):162-169.

55. Siegel JM. Narcolepsy: a key role for hypocretins (orexins). Cell. 1999;98(4):409-412.

56. Thannickal TC, Siegel JM, Nienhuis R, Moore RY. Pattern of hypocretin (orexin) soma and axon loss, and gliosis, in human narcolepsy. Brain pathology. 2003;13(3):340-351.

57. Gordon-Dseagu VL, Derkach A, Xiao Q, Williams I, Sampson J, Stolzenberg-Solomon RZ. The association of sleep with metabolic pathways and metabolites: evidence from the Dietary Approaches to Stop Hypertension (DASH) — sodium feeding study. Metabolomics.2019;15(4):48.

58. Minihane AM, Vinoy S, Russell WR, et al. Low-grade inflammation, diet composition and health: current research evidence and its translation. British Journal of Nutrition. 2015;114(7):999-1012.

59. Albenberg LG, Wu GD. Diet and the intestinal microbiome: associations, functions, and implications for health and disease. Gastroenterology. 2014;146(6):1564-1572. 
60. Katagiri R, Asakura K, Kobayashi S, Suga H, Sasaki S. Low intake of vegetables, high intake of confectionary, and unhealthy eating habits are associated with poor sleep quality among middle-aged female Japanese workers. Journal of occupational health. 2014:14-0051-OA.

61. Christian LM, Blair LM, Porter K, Lower M, Cole RM, Belury MA. Polyunsaturated fatty acid (PUFA) status in pregnant women: associations with sleep quality, inflammation, and length of gestation. PLoS One. 2016;11(2):e0148752.

62. Ma Q, Xing C, Long W, Wang HY, Liu Q, Wang R-F. Impact of microbiota on central nervous system and neurological diseases: The gut-brain axis. Journal of neuroinflammation. 2019;16(1):53.

63. Peuhkuri K, Sihvola N, Korpela R. Diet promotes sleep duration and quality. Nutrition research. 2012;32(5):309-319.

Table 1. The characteristics at baseline across quartiles of DII

\begin{tabular}{llllll}
\hline Characteristics (mean (SD) or \%) & DII quartiles & DII quartiles & DII quartiles & DII quartiles & DII quartiles \\
\hline & Q1 $(\mathrm{N}=62)$ & Q2 $(\mathrm{N}=62)$ & Q3 $(\mathrm{N}=63)$ & Q4 $(\mathrm{N}=62)$ & Total $(\mathrm{N}=249)$ \\
DII score & $-2.69 \pm 1.04$ & $-0.47 \pm 0.33$ & $0.57 \pm 0.26$ & $2.43 \pm 0.96$ & $-0.35 \pm 1.99$ \\
Age (years) & $25.50 \pm 4.82$ & $22.88 \pm 2.48$ & $23.34 \pm 3.95$ & $23.82 \pm 3.11$ & $23.88 \pm 3.81$ \\
Weight (kg) & $62.54 \pm 8.36$ & $56.60 \pm 7.18$ & $55.57 \pm 6.88$ & $51.19 \pm 8.26$ & $56.47 \pm 8.65$ \\
BMI (kg/m2) & $22.93 \pm 2.61$ & $21.73 \pm 2.20$ & $21.16 \pm 2.22$ & $20.04 \pm 2.97$ & $21.46 \pm 2.71$ \\
BMI categories (N) (\%) & & & & & \\
24.99 & $51(82.3)$ & $55(88.7)$ & $58(92.1)$ & $57(91.9)$ & $221(88.8)$ \\
25 & $11(17.7)$ & $7(11.3)$ & $5(7.9)$ & $5(8.1)$ & $28(11.2)$ \\
Education (N) (\%) & & & & & \\
Undergraduate & $37(59.7)$ & $44(71)$ & $48(76.2)$ & $49(79)$ & $178(71.5)$ \\
Other & $25(40.3)$ & $18(29)$ & $15(23.8)$ & $13(21)$ & $71(28.5)$ \\
WC (cm) & $81.82 \pm 7.55$ & $79.62 \pm 6.74$ & $79.19 \pm 9.21$ & $75.06 \pm 10.63$ & $78.92 \pm 8.95$ \\
WC. categories (N) (\%) & & & & \\
87.99 & $47(75.5)$ & $55(88.7)$ & $58(92.1)$ & $57(91.9)$ & $217(87.1)$ \\
88 & $15(24.2)$ & $7(11.3)$ & $5(7.9)$ & $8(8.1)$ & $32(12.9)$ \\
HC (cm) & $101.62 \pm 6.35$ & $98.38 \pm 5.39$ & $97.25 \pm 7.65$ & $95.56 \pm 6.35$ & $98.20 \pm 6.93$ \\
Height (cm) & $165.04 \pm 5.43$ & $161.23 \pm 4.94$ & $162.01 \pm 6.52$ & $159.75 \pm 5.32$ & $162.01 \pm 5.88$ \\
AVI & $13.50 \pm 2.48$ & $12.77 \pm 2.21$ & $12.71 \pm 3.34$ & $11.49 \pm 3.53$ & $12.61 \pm 3.02$ \\
BAI & $29.98 \pm 3.25$ & $30.06 \pm 2.47$ & $29.28 \pm 4.40$ & $29.38 \pm 3.38$ & $29.67 \pm 3.44$ \\
Conicity index & $1.22 \pm 0.08$ & $1.23 \pm 0.07$ & $1.24 \pm 0.09$ & $1.21 \pm 0.10$ & $1.22 \pm 0.09$ \\
P.A (met-min/week) & $1077.35 \pm 686.06$ & $950.56 \pm 495.67$ & $930.22 \pm 485.67$ & $896.70 \pm 490.82$ & $963.57 \pm 546.89$ \\
PSQI score & $6.01 \pm 3.83$ & $7.01 \pm 3.73$ & $7.53 \pm 3.53$ & $7.22 \pm 3.31$ & $6.95 \pm 3.63$ \\
PSQI score categories (N) (\%) & & & & & \\
4.99 & $26(41.9)$ & $17(27.4)$ & $11(17.5)$ & $12(19.4)$ & $66(26.5)$ \\
5 & $36(58.1)$ & $45(72.6)$ & $52(82.5)$ & $50(80.6)$ & $183(73.5)$ \\
\hline
\end{tabular}

1. Data are means $\pm \mathrm{SD}$ for quantitative variables and frequency (percent) for qualitative variables

a. From ANOVA for quantitative variables b. Chi-square for qualitative variables.

Abbreviation : Q; quartile, PSQI; Petersburg Sleep Quality Questionnaire, BMI; body mass index/z, WC; Waist circumference, HC; Hip circumference, DII; dietary inflammatory index, BAI; body adiposity index, AVI; abdominal volume index. Central obesity: WC [?] $88 \mathrm{~cm}$. Sleep status: good (PSQI score [?] 4.99) or poor (PSQI score [?] 5).

Table2. The mean +- SD of energy, macronutrient and micronutrient at baseline across quartiles of DII 


\begin{tabular}{|c|c|c|c|c|c|}
\hline Characteristics (mean (SD) or \%) & DII quartiles & DII quartiles & DII quartiles & DII quartiles & DII \\
\hline & $\mathrm{Q} 1(\mathrm{~N}=62)$ & $\mathrm{Q} 2(\mathrm{~N}=62)$ & Q3 $(\mathrm{N}=63)$ & $\mathrm{Q} 4(\mathrm{~N}=62)$ & Tot \\
\hline Energy (kcal/d) & $2243.26 \pm 299.95$ & $2030.09 \pm 257.84$ & $1993.32 \pm 246.90$ & $1836.02 \pm 296.28$ & 25 \\
\hline Carbohydrates intake (g) & $351.11 \pm 10.32$ & $291.46 \pm 11.35$ & $255.67 \pm 12.92$ & $228.01 \pm 12.29$ & 81. \\
\hline Protein intake $(\mathrm{g})$ & $66.71 \pm 10.32$ & $59.40 \pm 11.35$ & $57.55 \pm 47.57$ & $47.57 \pm 12.29$ & 7.8 \\
\hline Total fat intake (g) & $63.77 \pm 21.24$ & $70.21 \pm 18.94$ & $78.57 \pm 18.17$ & $81.73 \pm 20.03$ & 3.5 \\
\hline Saturated fats (g) & $19.41 \pm 9.09$ & $22.31 \pm 8.38$ & $27.13 \pm 10.03$ & $30.57 \pm 13.99$ & 4.8 \\
\hline Cholesterol (g) & $376.69 \pm 592.85$ & $511.68 \pm 559.21$ & $387.02 \pm 262.36$ & $342.14 \pm 323.80$ & 04 . \\
\hline Trance & $0.0014 \pm 0.0043$ & $0.0005 \pm 0.0018$ & $0.0015 \pm 0.0018$ & $0.0005 \pm 0.0013$ & .00 \\
\hline Vitamin B12 ( $\mu \mathrm{g})$ & $3.78 \pm 2.86$ & $4.21 \pm 3.36$ & $4.05 \pm 2.09$ & $3.16 \pm 1.76$ & 80 \\
\hline $\mathrm{Fe}(\mathrm{mg})$ & $20.02 \pm 5.23$ & $15.85 \pm 3.05$ & $14.31 \pm 2.96$ & $10.40 \pm 2.47$ & 5.1 \\
\hline Garlic $(\mathrm{g})$ & $0.09 \pm 0.28$ & $0.14 \pm 0.40$ & $0.26 \pm 0.44$ & $0.27 \pm 0.47$ & 19 \\
\hline Onion $(\mathrm{g})$ & $5.28 \pm 7.29$ & $7.54 \pm 8.57$ & $5.74 \pm 5.62$ & $6.93 \pm 7.13$ & 6.37 \\
\hline Monounsaturate & $22.20 \pm 8.09$ & $26.00 \pm 10.89$ & $30.68 \pm 12.32$ & $31.53 \pm 12.20$ & 27.6 \\
\hline Polyunsaturated fats (g) & $11.93 \pm 3.53$ & $10.97 \pm 3.27$ & $10.44 \pm 3.50$ & $8.29 \pm 2.65$ & 10.4 \\
\hline Fiber $(\mathrm{g})$ & $48.15 \pm 14.32$ & $39.06 \pm 15.45$ & $28.80 \pm 14.48$ & $20.40 \pm 9.12$ & 34.0 \\
\hline Caffeine (g) & $5.34 \pm 8.35$ & $11.13 \pm 12.53$ & $15.57 \pm 15.86$ & $23.65 \pm 23.05$ & 13.9 \\
\hline um (mg) & $332.21 \pm 83.30$ & $262.46 \pm 68.34$ & $225.50 \pm 62.36$ & $187.11 \pm 61.18$ & 51. \\
\hline Vitamin C (mg) & $241.74 \pm 127.58$ & $127.10 \pm 60.95$ & $78.69 \pm 43.02$ & $68.62 \pm 36.94$ & 28. \\
\hline Vitamin A $(\mu \mathrm{g})$ & $424.44 \pm 136.15$ & $294.92 \pm 193.69$ & $270.92 \pm 135.54$ & $185.97 \pm 112.84$ & 293. \\
\hline Vitamin E (mg) & $9.36 \pm 4.14$ & $7.79 \pm 3.92$ & $7.74 \pm 4.37$ & $6.35 \pm 3.62$ & 7.81 \\
\hline Beta carotene (units) & $2577.54 \pm 1626.29$ & $1420.50 \pm 963.19$ & $1141.26 \pm 686.92$ & $768.39 \pm 493.72$ & 1475 \\
\hline Folic acid $(\mu \mathrm{g})$ & $558.95 \pm 149.94$ & $441.59 \pm 100.38$ & $411.72 \pm 86.51$ & $310.90 \pm 99.71$ & 430. \\
\hline Vit D $(\mu \mathrm{g})$ & $0.47 \pm 0.31$ & $0.43 \pm 0.52$ & $0.38 \pm 0.40$ & $0.30 \pm 0.29$ & 0.39 \\
\hline Niacin (mg) & $22.56 \pm 4.55$ & $18.43 \pm 4.45$ & $17.17 \pm 4.47$ & $12.85 \pm 4.38$ & 17.7 \\
\hline Omega 3 fatty acids (g) & $0.15 \pm 0.18$ & $0.19 \pm 0.16$ & $0.11 \pm 0.08$ & $0.13 \pm 0.10$ & 0.15 \\
\hline Omega 6 fatty acids (g) & $10.20 \pm 3.30$ & $9.00 \pm 3.30$ & $8.65 \pm 3.81$ & $6.50 \pm 3.04$ & .59 \\
\hline Riboflavin (mg) & $1.94 \pm 0.60$ & $1.55 \pm 0.33$ & $1.63 \pm 0.36$ & $1.29 \pm 0.29$ & .60 \\
\hline Thiamin (mg) & $2.19 \pm 0.54$ & $1.64 \pm 0.41$ & $1.57 \pm 0.35$ & $1.15 \pm 0.32$ & 1.63 \\
\hline Zinc (mg) & $8.90 \pm 1.71$ & $7.17 \pm 1.45$ & $6.89 \pm 1.47$ & $5.42 \pm 1.84$ & 7.09 \\
\hline Selenium $(\mu \mathrm{g})$ & $117.66 \pm 47.70$ & $75.52 \pm 36.09$ & $73.74 \pm 29.62$ & $46.65 \pm 22.92$ & 78.3 \\
\hline B6 (mg) & $1.34 \pm 0.26$ & $1.08 \pm 0.24$ & $0.94 \pm 0.21$ & $0.72 \pm 0.19$ & 1.02 \\
\hline
\end{tabular}

The data are presented as "mean \pm SD".

*The significant difference based on One-way ANOVA $(\mathrm{p}<0.05)$.

\begin{tabular}{|c|c|c|c|c|c|c|c|c|}
\hline Variables & $\begin{array}{l}\text { Model } \\
0^{\mathrm{a}}\end{array}$ & $\begin{array}{l}\text { Model } \\
0^{\mathrm{a}}\end{array}$ & $\begin{array}{l}\text { Model } \\
0^{\mathrm{a}}\end{array}$ & $\begin{array}{l}\text { Model } \\
1^{\text {b }}\end{array}$ & $\begin{array}{l}\text { Model } \\
1^{\text {b }}\end{array}$ & $\begin{array}{l}\text { Model } \\
1^{\text {b }}\end{array}$ & $\begin{array}{l}\text { Model } \\
2^{\mathrm{c}}\end{array}$ & $\begin{array}{l}\text { Model } \\
2^{\mathrm{c}}\end{array}$ \\
\hline & $\begin{array}{l}\text { B } \\
\text { (Unstandard- } \\
\text { ized) }\end{array}$ & SE & $P$ - value & $\begin{array}{l}\text { B } \\
\text { (Unstandard- } \\
\text { ized) }\end{array}$ & SE & $P$ - value & $\begin{array}{l}\text { B } \\
\text { (Unstandard- }\end{array}$ & SE \\
\hline $\begin{array}{l}\text { Weight } \\
(\mathrm{kg})\end{array}$ & -2.04 & 0.24 & $\begin{array}{l}< \\
0.001\end{array}$ & 0.002 & 0.02 & 0.39 & 0.001 & 0.02 \\
\hline $\begin{array}{l}\mathrm{BMI} \\
\left(\mathrm{kg} / \mathrm{m}^{2}\right)\end{array}$ & -0.53 & 0.08 & $\begin{array}{l}< \\
0.001\end{array}$ & 0.04 & 0.04 & 0.36 & 0.04 & 0.04 \\
\hline $\begin{array}{l}\text { WC } \\
(\mathrm{cm})\end{array}$ & -1.33 & 0.27 & $\begin{array}{l}< \\
0.001\end{array}$ & 0.22 & 0.22 & 0.31 & 0.18 & 0.20 \\
\hline
\end{tabular}




\begin{tabular}{|c|c|c|c|c|c|c|c|c|}
\hline Variables & $\begin{array}{l}\text { Model } \\
0^{\mathrm{a}}\end{array}$ & $\begin{array}{l}\text { Model } \\
0^{\mathrm{a}}\end{array}$ & $\begin{array}{l}\text { Model } \\
0^{\text {a }}\end{array}$ & $\begin{array}{l}\text { Model } \\
1^{\mathrm{b}}\end{array}$ & $\begin{array}{l}\text { Model } \\
1^{\mathrm{b}}\end{array}$ & $\begin{array}{l}\text { Model } \\
1^{\mathrm{b}}\end{array}$ & $\begin{array}{l}\text { Model } \\
2^{\mathrm{c}}\end{array}$ & $\begin{array}{l}\text { Model } \\
2^{\mathrm{c}}\end{array}$ \\
\hline $\begin{array}{l}\mathrm{HC} \\
(\mathrm{cm})\end{array}$ & -1.17 & 0.20 & $\begin{array}{l}< \\
0.001\end{array}$ & 0.08 & 0.16 & 0.61 & 0.02 & 0.15 \\
\hline AVI & -0.4 & 0.09 & $\begin{array}{l}< \\
0.001\end{array}$ & 0.11 & 0.07 & 0.15 & 0.09 & 0.07 \\
\hline BAI & -0.17 & 0.10 & 0.11 & 0.11 & 0.11 & 0.33 & 0.08 & 0.11 \\
\hline $\begin{array}{l}\text { Conicity } \\
\text { index }\end{array}$ & -0.002 & 0.003 & 0.40 & 0.003 & 0.003 & 0.39 & 0.002 & 0.003 \\
\hline $\begin{array}{l}\text { PSQI } \\
\text { score } \\
\text { (ac- } \\
\text { cording } \\
\text { to con- } \\
\text { tinuous } \\
\text { DII) }\end{array}$ & 0.18 & 0.11 & 0.10 & 0.29 & 0.13 & 0.02 & 0.30 & 0.13 \\
\hline $\begin{array}{l}\text { PSQI } \\
\text { score } \\
\text { (according } \\
\text { to } \\
\text { quartiles } \\
\text { of the DII) }\end{array}$ & 0.41 & 0.20 & 0.04 & 0.61 & 0.22 & 0.008 & 0.60 & 0.23 \\
\hline
\end{tabular}

Table 3 . The Association between DII score (independent variables) with anthropometric variables and PSQI continuous score (dependent variables)

a. Model 0, linear regression analysis without adjustment; b. Model I, linear regression analysis with adjustment for energy intake; c. Model II, linear regression analysis with correction for age, energy intake, physical activity, and education

Abbreviation : BMI; body mass index, DII; dietary inflammatory index, BAI; body adiposity index, AVI; abdominal volume index; PSQI; Petersburg Sleep Quality Questionnaire

\begin{tabular}{|c|c|c|c|c|}
\hline Variable & $\mathrm{Q} 1(\mathrm{~N}=62)$ & $\mathrm{Q} 2(\mathrm{~N}=62)$ & $\mathrm{Q} 3(\mathrm{~N}=63)$ & $\mathrm{Q} 4(\mathrm{~N}$ \\
\hline \multicolumn{5}{|l|}{ BMI.cat ( according to quartiles of the DII) } \\
\hline Model $0^{\mathrm{a}}$ & 1 (ref) & $2.45(0.80-7.55)$ & $1.45(0.43-4.84)$ & 0.98 \\
\hline Model $1^{\mathrm{b}}$ & 1 (ref) & $0.2 \mathrm{x}(0.03-1.17)$ & $0.93(0.13-6.69)$ & 0.86 \\
\hline Model $2^{\mathrm{c}}$ & 1 (ref) & $0.18(0.03-1.12)$ & $0.41(0.04-3.64)$ & $0.57(0$ \\
\hline \multicolumn{5}{|c|}{ Central obesity ( according to quartiles of the DII) } \\
\hline Model $0^{\mathrm{a}}$ & 1 (ref) & $3.63(1.23-10.74)$ & $1.45(0.43-4.84)$ & 0.98 \\
\hline Model $1^{\mathrm{b}}$ & 1 (ref) & $0.57(0.13-2.46)$ & $0.76(0.14-4.01)$ & 0.69 \\
\hline Model $2^{\mathrm{c}}$ & 1 (ref) & $0.30(0.03-3.06)$ & $3.80(0.32-44.67)$ & $3.15(0$ \\
\hline \multicolumn{5}{|l|}{ Sleep.cat (quartiles of the DII) } \\
\hline Model $0^{\mathrm{a}}$ & 1 (ref) & $0.33(0.14-0.74)$ & $0.63(0.27-1.47)$ & $1.13(0$ \\
\hline Model $1^{\mathrm{b}}$ & 1 (ref) & $0.31(0.12-0.78)$ & $0.61(0.26-1.47)$ & $1.11(0$ \\
\hline Model $2^{\mathrm{c}}$ & 1 (ref) & $0.30(0.12-0.78)$ & $0.61(0.25-1.48)$ & $1.13(0$ \\
\hline Sleep.cat (according to continuous DII) & B (unstandardized) & $\mathrm{SE}$ & OR & CI \\
\hline Model $0^{\mathrm{a}}$ & 0.19 & 0.07 & 1.21 & $1.05-1$ \\
\hline Model $1^{\mathrm{b}}$ & 0.19 & 0.08 & 1.21 & $1.03-1$ \\
\hline Model $2^{\mathrm{c}}$ & 0.19 & 0.08 & 1.22 & $1.03-1$ \\
\hline
\end{tabular}


Table 4 . Odds rations (95\%CI) for obesity and sleep quality (dependent variables) according to DII score (independent variables)

* $P<0.05$ statistically significant by Multivariable Logistic Regression. a . Model 0: unadjusted. b . Model 1: adjusted for energy intake. c . Model 2: adjustment for age, education, physical activity, energy intake.

Central obesity: obese (WC [?] $88 \mathrm{~cm}$ ) or normal (WC[?] 87.99)

BMI categories: normal (BMI [?] $24.99 \mathrm{~kg} / \mathrm{m}^{2}$ ) or overweight (BMI [?] $25 \mathrm{~kg} / \mathrm{m}^{2}$ )

Sleep categories: good (sleep score [?] 4.99) or poor (sleep score [?] 5.00) 